\title{
Bibliothek des Jahres
}

\section{Irina Sens*, Sören Auer, Thomas Bähr, Sandra Niemeyer, Margret Plank und Philip Schrenk Die TIB: Mehr als eine Bibliothek}

https://doi.org/10.1515/bfp-2020-2040

Zusammenfassung: Die TIB gestaltet aktiv den digitalen Wandel von Bibliotheken mit - in Form von Open Science, Open Access, Forschungsdaten und digitaler Langzeitarchivierung sowie bei der Entwicklung von Diensten an der Schnittstelle von analogen und digitalen Formaten. Sie baut ihre Rolle als deutsches Informationszentrum für die Digitalisierung von Wissenschaft und Technik in Zukunft weiter aus und setzt auf die Entwicklung neuer digitaler Angebote, die klassische bibliothekarische Dienste und innovative Forschungsergebnisse miteinander verzahnen.

Als wissenschaftliche Infrastruktureinrichtung steht sie vor zwei großen Herausforderungen: Als Deutsche Zentrale Fachbibliothek für Technik und Naturwissenschaften bewegt sie sich als Akteurin im Zentrum des digitalen Wandels des wissenschaftlichen Arbeitens, des digitalen Publikationswesens und der Globalisierung von Information und Kommunikation. Gleichzeitig erbringt sie als von Bund und Ländern finanzierte Einrichtung verstärkt eigene Forschung.

Schlüsselwörter: Data Science; Open Research Knowledge Graph; Forschungsdaten; Dokumentlieferung; Langzeitarchivierung; Open Access

\section{The TIB - More than Just a Library}

Abstract: TIB plays an active role in achieving the digital transformation of libraries - not only in the form of open science, open access, research data, and digital preservation, but also in the development of services at the interface between analogue and digital formats. The library will continue to expand its role as the German information centre for the digitalisation of science and technology in the future, focusing on the development of new digital offerings that link traditional library services with innovative research results.As a scientific infrastructure facility, TIB faces two major challenges: being the German national

\footnotetext{
*Kontaktperson: Dr. Irina Sens, irina.sens@tib.eu

Prof. Dr. Sören Auer, auer@tib.eu

Thomas Bähr, thomas.baehr@tib.eu

Dr.Sandra Niemeyer, sandra.niemeyer@tib.eu

Margret Plank, margret.plank@tib.eu

Philip Schrenk, philip.schrenk@tib.eu
}

library of science and technology, it operates as a stakeholder at the heart of the transition to the digital production of scientific work, digital publishing, and the globalisation of information and communication. At the same time, TIB increasingly conducts its own research as an institute financed by the German federation and the federal states.

Keywords: Data Science; Open Research Knowledge Graph; research data; document delivery; digital preservation; Open Access

\section{Inhalt}

1 Die TIB als Deutsche Zentrale Fachbibliothek . . 475

1.1 Open-Access-Beratung und Open-Access-Publikationsfonds. . . . . . . . . . . . . 476

1.2 Nachhaltige Informationsinfrastruktur . . . . . . 476

1.3 Graue Literatur . . . . . . . . . . . . . . . . . . . . 477

2 Die TIB als Universitätsbibliothek . . . . . . . . . 477

3 TIB als Forschungseinrichtung - Wandel zum Informationszentrum für die Digitalisierung . . . 479

4 Produkte, Services und Angebote - eine Auswahl. . . . . . . . . . . . . . . . . . . 479

4.1 Digitale Langzeitarchivierung . . . . . . . . . . . 479

4.2 Open-Access-Transformation . . . . . . . . . 480

4.3 Lizenzierung elektronischer Ressourcen. . . . . . 480

4.4 Das TIB AV-Portal - eine verlässliche Infrastruktur für wissenschaftliche Filme . . . . . . . . . 481 4.5 Digitale Formate . . . . . . . . . . . . . . 481

5 Wo geht es hin?................ 482

5.1 Forschungsdaten und Nationale Forschungsdateninfrastruktur (NFDI) . . . . . . . . . . . . . 482

5.2 Open Research Knowledge Graph (ORKG) . . . . . 482

5.3 Organisation von Daten und Wissen zu Covid-19 . 483

6 Schlusswort .................... 483

Wissen und Informationen frei verfügbar machen, teilen und kulturelles Erbe bewahren: Die Aufgaben der TIB Leibniz-Informationszentrum Technik und Naturwissenschaften und Universitätsbibliothek sind umfangreich. Funktion und Aufgaben der TIB fußen dabei auf drei Säulen: Sie ist Deutsche Zentrale Fachbibliothek für Technik sowie Architektur, Chemie, Informatik, Mathematik und 
Physik, sie fungiert für die Leibniz Universität Hannover als Universitätsbibliothek, und als Forschungseinrichtung betreibt sie angewandte Forschung und Entwicklung, um neue Dienstleistungen $\mathrm{zu}$ realisieren und bestehende $\mathrm{zu}$ verbessern.

Die heutige TIB, die Teil der Leibniz-Gemeinschaft ist, geht aus der 1831 gegründeten Bibliothek der im selben Jahr errichteten „Höheren Gewerbeschule zu Hannover“ und der 1959 gegründeten „Technischen Informationsbibliothek" hervor. Seit 2016 ist die Bibliothek eine Stiftung des öffentlichen Rechts des Landes Niedersachsen.

\section{Die TIB als Deutsche Zentrale Fachbibliothek}

Mit der Gründung der Technischen Informationsbibliothek - kurz TIB - im Jahr 1959 als erste von dann vier zentralen Fachbibliotheken wurde der Tatsache Rechnung getragen, dass die Literaturversorgung für die Ingenieurwissenschaften in Deutschland, insbesondere für die Industrie, nicht ausreichend war. Verbunden wird dies stets mit dem Sputnik-Schock, dem technologischen Vorsprung der Sowjetunion gegenüber den westlichen Industrienationen. Dieser führte dazu, dass

a) der Zugang zu osteuropäischer und ostasiatischer Literatur verbessert werden musste und

b) es eine zentrale Bibliothek für Technik und Ingenieurwissenschaften geben sollte - und nicht wie bisher ein dezentrales System.

Die Standortwahl für die TIB fiel auf Hannover. Die Hauptwettbewerberin, die Bibliothek des Deutschen Museums in München, die vor dem Krieg diese Funktion quasi innegehabt hatte, hatte keine Anbindung an eine Universität. Darüber hinaus hatte die Universitätsbibliothek Hannover ihren Bestand über den Krieg retten können.

Am 15. Juni 1959 wurde per Erlass die Technischen Informationsbibliothek gegründet: Bewilligt wurden sieben zusätzliche Stellen sowie 100000 DM Sachmittel. Die Bibliothek befand sich im Welfenschloss, Gründungsdirektor war Dr. Wilhelm Grunwald.

Von Beginn an standen also die Dokumentlieferung ${ }^{1}$ an akademische und kommerzielle Kunden sowie die Beschaffung der Literatur aus Osteuropa und Ostasien im Fokus. 1977 war die TIB größte Direktlieferantin in Deutschland mit 332000 Bestellungen. Im selben Jahr wurde die Fi-

1 https://tib.eu/tib-dokumentlieferung.

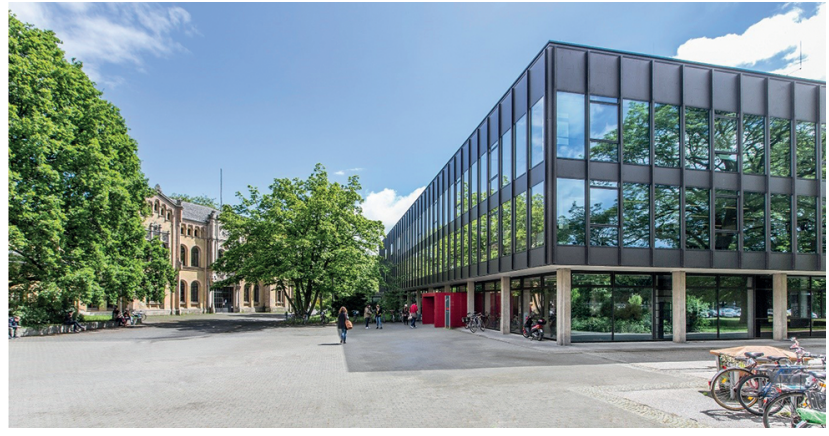

Abb. 1: Außenansicht des TIB-Standorts Technik/Naturwissenschaften und des Marstallgebäudes ${ }^{2}$

nanzierung der TIB durch die Deutsche Forschungsgemeinschaft (DFG) auf die Bund-Länder-Finanzierung umgestellt. In den 1990er-Jahren beginnen mit der TIBQuickSerie die Beschleunigung und Elektrifizierung der Direktbestellungen durch die TIB - nachdem sie 1981 die erste Bibliothek weltweit war, die Eilbestellungen per Fax auslieferte. Der Erfolg der Dokumentlieferung bedingte wohl auch die Klage des Börsenvereins bezüglich der Erstellung der Kopien auf Einzelbestellung. 1999 gewann die TIB diesen Prozess vor dem Bundesgerichtshof.

Trotz der zunehmenden Verfügbarkeit elektronischer Ressourcen - bedingt durch das Förderprogramm der DFG für National- und Allianzlizenzen sowie des steigenden Anteils an Open Access - ist die TIB-Dokumentlieferung nach wie vor ein wesentlicher Bestandteil der Aufgaben der Bibliothek. Sie versorgt ihre Kundinnen und Kunden aus Wissenschaft und Industrie über die Dokumentlieferung schnellstmöglich und umfassend mit den benötigten Fachinformationen. Basis dafür ist der in Breite und Tiefe exzellente, nahezu vollständige Bestand innerhalb der TIB-Fachgebiete Technik sowie Architektur, Chemie, Informatik, Mathematik und Physik. Im Jahr 2019 lieferte die TIB knapp 164000 Direkt- und mehr als 36000 Leihverkehrbestellungen aus. Das Portfolio umfasst dabei die traditionelle Fernleihe, die Dokumentlieferung unter anderem über das TIB-Portal ${ }^{3}$ und subito ${ }^{4}$ sowie den Einzelartikelverkauf (Single Article Sales). Hier können die Kundinnen und Kunden sofort auf das elektronische Dokument zugreifen, wobei das TIB-Portal die Anfrage beim Verlag auslöst und damit als neutraler Anbieter agiert.

2 (C) TIB/C. Bierwagen.

3 https://www.tib.eu/de/.

4 https://www.subito-doc.de/. 
Neu im Bestand

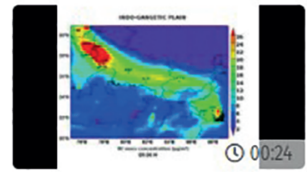

BC-animation-1

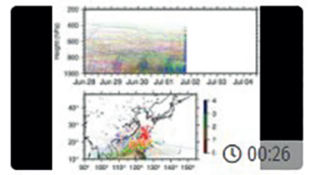

Trajectories animation

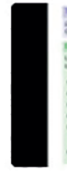

Gröbner bases over K and $\mathrm{Z}$ in theory and

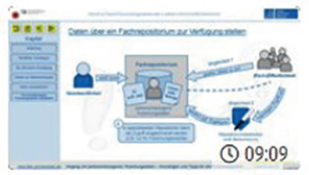

Personenbezogene Forschungsdaten -

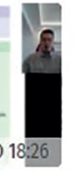

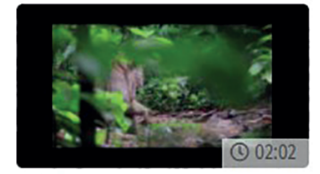

Infanticide in Macaca fascicularis

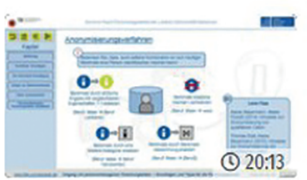

Personenbezogene Forschungsdaten -

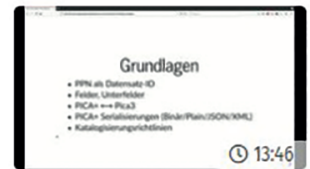

PICA-Formate entschlüsseln mit Avram

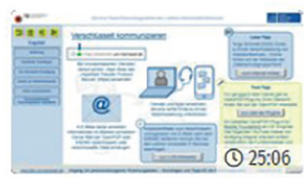

Personenbezogene Forschungsdaten -

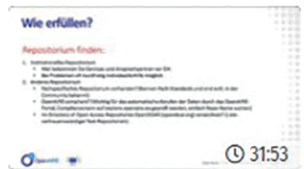

Open-Access-Vorgaben in EU-Projekten

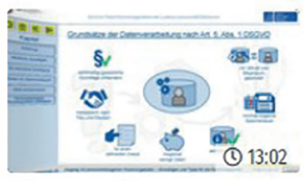

Personenbezogene Forschungsdaten -

Abb. 2: Das AV-Portal der TIB

\subsection{Open-Access-Beratung und Open-Access-Publikationsfonds}

Die TIB berät Autorinnen und Autoren zu ihren Rechten und Möglichkeiten rund um das Thema Open-AccessVeröffentlichungen und unterstützt sie bei ihren Publikationsentscheidungen. Dazu gehören Schulungsangebote vor Ort, Informationsveranstaltungen in anderen Einrichtungen sowie Webinare rund um Open Access und wissenschaftliches Publizieren. Die TIB informiert außerdem über ihre Angebote zur Übernahme von OpenAccess-Publikationskosten. Über den Open-Access-Publikationsfonds der Leibniz Universität Hannover stellt sie als Universitätsbibliothek den Wissenschaftlerinnen und Wissenschaftlern finanzielle Mittel für Publikationskosten von Open-Access-Veröffentlichungen bereit. Über den OpenAccess-Publikationsfonds der Leibniz-Gemeinschaft haben die Mitglieder der beteiligten Leibniz-Einrichtungen zudem die Möglichkeit, ihre Artikel in Open-Access-Zeitschriften zu finanzieren. Zur Stärkung offener Publikationsformen fördert das Bundesministerium für Bildung und Forschung (BMBF) das Projekt open-access.network. ${ }^{5}$ Neben der Schaffung einer Plattform als zentralem Ort der

5 https://open-access.net/ueber-uns/oa-network.
Information und des Austauschs steht vor allem der Aufbau von Kompetenzen im Fokus der Arbeit. Die TIB übernimmt hier Arbeitsschwerpunkte bei der Erstellung von Informations- und Lehrmaterialien, bei der Konzeption und Durchführung von Online-Schulungen, bei der Erforschung von Open-Access-Lücken und beim Betrieb eines zentralen Diskussionsforums.

\subsection{Nachhaltige Informationsinfrastruktur}

Wissen und Informationen müssen nachhaltig und weiternutzbar sein - das ist aber nur mit nachhaltigen Informationsinfrastrukturen möglich. Als weltweit erste Registrierungsagentur für DOIs (Digital Object Identifier) für wissenschaftliche Objekte wie Forschungsdaten, Software, 3D-Objekte, wissenschaftliche Videos und vieles mehr setzt sich die TIB bereits seit 2004 für die Nachhaltigkeit verschiedener wissenschaftlicher Objekte ein. Im Bereich Langzeitarchivierung sorgt die TIB dafür, dass kulturelles Erbe bewahrt und somit nachhaltig verfügbar ist. So macht sie beispielsweise im Projekt Delft ${ }^{6}$ Filmschätze aus der Ethnologie zugänglich. Die fast 2000 historisch wertvollen

6 http://tib.eu/delft. 
Filme zeigen kulturelle Bräuche und handwerkliche Traditionen, die zum Teil bereits aus unserem Alltag verschwunden sind oder sich über die Jahre stark verändert haben. Einige der Filme sind mehr als 100 Jahre alt, die jüngsten stammen aus den 1980er-Jahren. Die Mehrzahl der Filme ist Teil der „Encyclopaedia Cinematographica“. Die TIB digitalisiert, erschließt und stellt sie - soweit rechtlich möglich - online in ihrem AV-Portal ${ }^{7}$ bereit. Das eröffnet Interessierten aus Forschung und Lehre ganz neue Möglichkeiten, mit diesem wertvollen und einmaligen kulturellen Erbe zu arbeiten.

\subsection{Graue Literatur}

Graue Literatur - häufig definiert als Veröffentlichungen, die nicht im Buchhandel erhältlich sind - ist oft schwer beschaffbar. Graue Literatur, etwa in Form von Konferenzund Forschungsberichten, Gutachten oder auch Diplomarbeiten, beinhaltet wertvolles Wissen, das eine wichtige Informationsquelle für Wissenschaft und Forschung ist. Konferenzberichte enthalten beispielsweise häufig den neuesten Stand eines Forschungsbereichs.

Die graue Literatur ${ }^{8}$ ist ein Spezialgebiet der TIB. Sie erwirbt und erschließt graue Literatur in den ingenieurwissenschaftlichen Fächern sowie Architektur, Chemie, Informatik, Mathematik und Physik und stellt sie Wissenschaft, Forschung und Wirtschaft zur Benutzung bereit. Dazu zählen Konferenzveröffentlichungen weltweit, deutsche und ausländische Forschungsberichte, ostasiatische und osteuropäische Zeitschriften und Monografien, Hochschulschriften sowie Patentschriften und Normen. Zudem ist die TIB Depotbibliothek für Abschlussberichte öffentlich geförderter Vorhaben verschiedener Bundesministerien wie dem Bundesministerium für Bildung und Forschung (BMBF), dem Bundesministerium für Wirtschaft und Energie (BMWi) und dem Bundesministerium für Ernährung und Landwirtschaft (BMEL) - sowie zahlreicher anderer Institutionen und Forschungseinrichtungen.

Um graue Literatur sichtbarer zu machen, unterstützt die TIB zudem seit 2015 die von der TIB ins Deutsche übersetzten Forderungen der Pisa Declaration:' Regierungen und Organisationen sollen graue Literatur stärken. Darin wird gefordert, dass sie sich stärker für Open Access engagieren, hochwertige graue Literatur stärker anerkennen

7 https://av.tib.eu/.

8 http://tib.eu/graue-literatur.

9 https://www.tib.eu/fileadmin/Daten/dokumente/recherchieren-e ntdecken/Pisa-Declaration-deutsche-UEbersetzung.pdf. und die Bestandsentwicklung sowie die Langzeitarchivierung grauer Literatur stärker unterstützen.

\section{Die TIB als Universitätsbibliothek}

Im Kern geht die Universitätsbibliothek Hannover auf die Bibliothek der 1831 gegründeten Höheren Gewerbeschule in Hannover zurück und folgt in ihrer Funktion deren Entwicklung über die Polytechnische Schule (ab 1847), die Technische Hochschule (ab 1879) und die Technische Universität (ab 1968) zur Universität (seit 1978 mit Integration der Pädagogischen Hochschule). ${ }^{10}$ Seit Errichtung der Stiftung „Technische Informationsbibliothek (TIB)“ zum 1. Januar 2016 existiert die Universitätsbibliothek Hannover nicht mehr als Zentrale Einrichtung der Leibniz Universität Hannover in organisatorischem Sinn, sondern nur noch als Funktion der nunmehr rechtlich selbstständigen TIB. Das „Gesetz über die Stiftung ,Technische Informationsbibliothek (TIB)““11 legt in § 2 (3) fest, dass die „TIB den Betrieb der Universitätsbibliothek der Universität Hannover [übernimmt] und [...] die vom Land Niedersachsen finanzierte Literatur- und Informationsversorgung der Universität Hannover [gewährleistet]“. Die Universität Hannover hat damit ihre Literaturversorgung an die TIB ,ausgelagert', was jedoch keineswegs eine Wertung impliziert, denn schon zuvor gab es hier organisatorische Besonderheiten.

Für die Literaturversorgung der Universität Hannover war bis Ende 2002 aber nicht nur die „Universitätsbibliothek und Technische Informationsbibliothek (UB/TIB)“ zuständig, wie die heutige TIB damals hieß. Während die TIB für die technisch-naturwissenschaftlichen Fächer verantwortlich war, übernahm für die geistes- und sozialwissenschaftlichen Fächer auch die Niedersächsische Landesbibliothek (NLB) die Versorgung. ${ }^{12}$ Sowohl die UB/TIB als auch die NLB unterhielten zu diesem Zweck mehrere Fachbereichsbibliotheken, von denen die Fachbereichsbibliothek Erziehungswissenschaften bei der NLB aus der Bibliothek der ehemaligen Pädagogischen Hochschule entstanden ist.

Im Zuge einer Neuordnung der universitären Literaturversorgung am Standort Hannover wurden zum 1. Januar 2003 die Fachbereichsbibliotheken der NLB mit ihren Be-

10 Zur Entwicklung bis dahin vgl. Schlitt (1981) mit weiteren Literaturhinweisen.

11 Vom 14. Juli 2015 (Nds. GVBl. Nr. 10/2015, 151), geändert durch Art. 4 des Gesetzes vom 15. Dezember 2015 (Nds. GVBl. Nr. 22/2015, 384) und Art. 13 des Gesetzes v. 20.12.2016 (Nds. GVBl. 20/2016, 308). 12 Ausführlicher dazu Vollers (1983) sowie Vollers (1995). 
ständen und ihrem Personal der UB/TIB zugeordnet. Da die Bibliotheken in Hannover mit dem Hannoverschen Online-Bibliothekssystem HOBSY ${ }^{13}$ seit 1993 ein gemeinsames Lokales Bibliothekssystem mit einheitlichem Bibliotheksausweis nutzen und insbesondere in Fragen der Benutzung eng kooperieren, verlief diese Änderung für die Benutzerinnen und Benutzer weitgehend reibungslos. ${ }^{14}$

Die UB/TIB bestand räumlich bis dahin aus dem Hauptstandort am Welfengarten, einem Außenmagazin sowie drei Fachbereichsbibliotheken (mit den Fächern Gartenbau, Berufsdidaktik und Physik). Hinzu kamen von der NLB die Fachbereichsbibliotheken am Königsworther Platz (Wirtschaftswissenschaften, Rechtswissenschaften, Literatur- und Sprachwissenschaften), die Fachbereichsbibliothek Erziehungswissenschaften (ehemals Bibliothek der Pädagogischen Hochschule), die Fachbereichsbibliothek Sozialwissenschaften sowie die Bereichsbibliothek Geschichte und Religionswissenschaft.

Die Entwicklung seit 2003 war zunächst bestimmt von der organisatorischen Integration der ehemaligen Fachbereichsbibliotheken der NLB. Als Randbedingungen waren Anforderungen an Personaleinsparung, räumliche Prioritätensetzungen in der Universität und nicht zuletzt die Neuordnung der Lehrerausbildung (mit Verlagerung eines großen Teils der Lehrerausbildung von Hannover nach Hildesheim) im Rahmen des Hochschuloptimierungskonzepts der niedersächsischen Landesregierung ${ }^{15}$ zu berücksichtigen. Aus diesen Gründen wurden die Fachbereichsbibliothek Physik und Gartenbau aufgelöst und die Bestände in Lesesäle und Magazin integriert sowie mit Aufgabe des Standorts Bismarckstraße (frühere Pädagogische Hochschule) auch die Fachbereichsbibliothek Erziehungswissenschaften aufgelöst und als Fachlesesaal in die Fachbereichsbibliotheken am Königsworther Platz integriert. $\mathrm{Zu}$ diesem Zweck wurde dort - im laufenden Betrieb - ein fünftes Obergeschoss errichtet, das zugleich als Keimzelle der Modernisierung des Lernraums an diesem Standort wurde.

Die verringerte Zahl der Standorte bot die Chance für Vereinheitlichung von Benutzungsbedingungen und Ausweitung von Öffnungs- und Servicezeiten. Die Modularisierung der Studiengänge führte zu quantitativen und qualitativen Veränderungen bei Auskunft und Schulungen, erhöhter Nachfrage nach Lehrbüchern, vor allem aber zu wachsendem Bedarf an Lernräumen, deren Nutzung sich zunehmend von Bibliotheksbeständen entkoppelte.

13 https://www.hobsy.de/.

$14 \mathrm{Zu}$ HOBSY vgl. ausführlicher Tempel (2020).

15 https://www.mwk.niedersachsen.de/startseite/aktuelles/pres seinformationen/-17917.html.
Welchen Weg die Universitätsbibliothek hier zurückgelegt hat, wird schon deutlich beim Vergleich nur der drei Kennzahlen Öffnungstage, Öffnungsstunden pro Woche und Benutzerarbeitsplätze. Hatte die TIB 2003 an ihren Standorten noch bis zu 298 Tage im Jahr bis zu 58 Stunden pro Woche geöffnet und bot 1281 Benutzerarbeitsplätze, waren es 2019 schon 349 Öffnungstage (durch die Sonntagsöffnung am TIB-Standort Conti-Campus) mit bis zu 102 Öffnungsstunden pro Woche und einem Angebot von 1827 Benutzerarbeitsplätzen. Möglich wurden diese Erweiterungen auch durch die Mittel aus Studienbeiträgen in Niedersachsen und, seit Abschaffung dieser Beiträge, deren Kompensation in Form von Studienqualitätsmitteln. Das ursprüngliche Ziel war es, während der gesamten Öffnungszeiten auch den vollen Service anzubieten (Leihstelle mit Kasse und Anmeldung, Auskunft, Bereitstellung aus dem Magazin). Über viele Jahre war dies auch möglich, sodass an den Standorten Technik/Naturwissenschaften und Conti-Campus an fünf Wochentagen der volle Service von 9 bis 22 Uhr geboten wurde und am Samstag von 9 bis 18 Uhr. Bei Einführung der Sonntagsöffnung, Erweiterung samstags bis 22 Uhr und Öffnung Montag bis Freitag um 8 beziehungsweise 9 Uhr blieb es jedoch bei den bisherigen Servicezeiten. Es wurde ein Betrieb mit Hilfskräften, unterstützt durch Wachdienst, eingeführt. Anlässlich der erneuten Ausweitung der Öffnungszeiten am Standort ContiCampus, nämlich Montag bis Freitag bis $24 \mathrm{Uhr}$, wurden die Servicezeiten überprüft. Es zeigte sich, dass es reicht, den vollen Service nur noch bis 20 Uhr anzubieten und danach die Lernraumnutzung mit Basisdienstleistungen wie Ausleihe und Rücknahme zu ermöglichen.

Zwischen TIB als Universitätsbibliothek und als Zentraler Fachbibliothek gab es von Anfang an Synergieeffekte. Die technisch-naturwissenschaftlichen Fächer der Universität konnten stets auf Bestände der TIB zugreifen und hatten damit eine tiefere Literaturversorgung vor Ort als eine Universitätsbibliothek normalerweise leisten könnte. Erwerbungsmittel für die Universitätsbibliothek können eingesetzt werden für das, was die TIB nicht erwirbt oder lizenziert. Dazu gehört unter anderem die Finanzierung von Campuslizenzen für Zeitschriften, Datenbanken und E-Books, die die TIB als Zentrale Fachbibliothek ihren Kundinnen und Kunden nicht zur Verfügung stellen kann.

Die Frage nach Synergieeffekt oder Reibungsverlust stellt sich in besonderem Maße bei den Webauftritten der TIB. Die Bedürfnisse der beiden großen, in sich schon heterogenen und untereinander weitgehend disjunkten Zielgruppen der Benutzerinnen und Benutzer vor Ort (Funktion der Universitätsbibliothek) und der überregionalen Kundinnen und Kunden (Funktion der Zentralen Fachbibliothek) mit einer Webseite und einem Rechercheportal 
abzudecken, ist eine Optimierungsaufgabe, die die TIB schon lange beschäftigt und weiterhin beschäftigen wird.

\section{TIB als Forschungseinrichtung - Wandel zum Informationszentrum für die Digitalisierung}

Die TIB treibt die Entwicklung zum Informationszentrum für die Digitalisierung von Technik und Naturwissenschaften konsequent voran und unterstützt die verschiedenen Phasen der Forschung in Wissenschaft und Wirtschaft. Ihre Forschungsschwerpunkte liegen auf Data Science \& Digital Libraries, wissenschaftlichem Datenmanagement, nicht-textuellen Materialien, Open Science und Visual Analytics. Dazu wird der etablierte Innovationszyklus zum Transfer von Forschungsergebnissen in potenzialträchtige Anwendungen weiterentwickelt. Im Fokus steht insbesondere die Verzahnung der Forschungsaktivitäten mit dem Bibliotheksbetrieb.

Die Forschungsaktivitäten sollen insbesondere Fragen beantworten wie: Wie kann in großen Datenbeständen im Internet, in Bibliotheken oder Archiven optimal gesucht werden? Wie lassen sich verteilte Informationen besser vernetzen und Informationsflüsse zwischen Forschenden reibungsloser organisieren? Ziel des Bereiches Forschung und Entwicklung an der TIB ist es somit, einen innovativen, effizienten und nahtlosen Zugriff auf Daten, Informationen und Wissen zu ermöglichen. Aktuell arbeiten mehr als 70 Mitarbeiterinnen und Mitarbeiter in diesem Bereich, circa die Hälfte davon im Rahmen von geförderten Drittmittelprojekten.

Die Aktivitäten erfolgen überwiegend in Form von Projekten - auf nationaler, europäischer und internationaler Ebene - und umfassen folgende Forschungsgruppen und Lab-Bereiche:

- Forschungsgruppe Data Science \& Digital Libraries

- Forschungsgruppe Scientific Data Management

- Forschungsgruppe Visual Analytics

- Open Science Lab

- Lab Nicht-Textuelle Materialien (NTM)

- Bereich Software-Entwicklung

Drittmittelgeförderte Forschungs- und Entwicklungsvorhaben beinhalten häufig transferrelevante Fragestellungen und werden stets mitgedacht. Der Transfer von Forschungsergebnissen in relevante Dienste und Anwendungen für die TIB-Nutzergruppen wird durch eine enge Verzahnung mit den bibliothekarischen Bereichen der Bibliothek gewährleistet.

\section{Produkte, Services und Angebote - eine Auswahl}

\subsection{Digitale Langzeitarchivierung}

Seit zehn Jahren betreibt die TIB gemeinsam mit ihren Partnerbibliotheken ZB MED - Informationszentrum Lebenswissenschaften und ZBW - Leibniz-Informationszentrum Wirtschaft ein kooperatives System zur digitalen Langzeitarchivierung (dLZA). Die TIB stellt ihren Partnern hierfür die erforderlichen technischen und infrastrukturellen Rahmenbedingungen als Dienstleistung bereit. Als Basis dient dabei das Langzeitarchivierungssystem Rosetta ${ }^{16}$ der Firma Ex Libris. Derzeit werden rund sechs Millionen Objekte in circa 170 verschiedenen Formaten archiviert.

Neben dem Aufbau einer leistungsstarken und skalierbaren technischen Infrastruktur sowie der Marktanalyse und anschließender Auswahl einer geeigneten Software ist der Aufbau von Know-how und die konsequente Weiterentwicklung von Wissen über die dLZA ein entscheidendes Erfolgskriterium. Voraussetzung dafür ist die Definition, was unter dLZA verstanden wird. Die TIB versteht darunter sowohl die Erhaltung des Datenstroms (Bitstream-Preservation) als auch die Erhaltung von Informationen auf der Format- und inhaltlichen Ebene. Objektbezogene Fragestellungen bilden dabei den Ausgangspunkt jedweder Erhaltungsmaßnahme: Welchen Erhaltungswert haben die Objekte? Wer ist dafür verantwortlich? Welche Nutzungsszenarien gibt es? In welchem Zustand sind die Objekte? Welche Informationen liegen darüber vor? Das Wissen um die Objekte in Form von Metadaten, ihre Provenienz sowie die rechtlichen Rahmenbedingungen sind die Voraussetzung, um erfolgreiche Erhaltungsstrategien zu entwickeln und umzusetzen.

Eine weitere Voraussetzung für eine erfolgreiche dLZA ist die Dokumentation aller Prozessschritte innerhalb des LZA-Systems wie auch die gewissenhafte Dokumentation aller externen Prozesse sowie die übergeordnete Beschreibung der dLZA als strategische Säule der TIB. Bei der ersten Aufgabe werden mithilfe administrativer Metadaten die Informationen $\mathrm{zu}$ den Objekten archiviert, die eine Aussage über deren Provenienz, die Arbeitsschritte innerhalb des Systems und deren Ergebnisse enthalten. Sie enthalten auch Informationen darüber, wer an den Objekten warum gearbeitet hat und unter welchen Voraussetzungen sie in das LZA-System aufgenommen wurden.

16 https://www.exlibrisgroup.com/de/produkte/rosetta/. 
Die zweite Form der Dokumentation geschieht im Idealfall in Form von Policies. Diese beschreiben nicht die Objekte im Einzelnen, sondern geben Auskunft darüber, welche Art von Kollektion aus welchem Grund auf welchem Weg unter welchen (rechtlichen) Bedingungen archiviert werden soll. Sie beschreiben weiterhin die Struktur der Objekte innerhalb einer Sammlung und die Anforderungen der Dateneigentümer und ihrer Nutzerinnen und Nutzer an die Objekte. Darin wird verbindlich geregelt, welche Verpflichtungen das Langzeitarchiv zur Erhaltung der Daten übernimmt und wie die Aufgaben und Verantwortungen zwischen den Datenproduzentinnen und -produzenten und dem Langzeitarchiv verteilt sind.

Die dLZA kein einmaliger Vorgang ist, sondern ein Prozess von sich wiederholenden Aufgaben. Aufgrund der sich ständig verändernden Daten, ihrer technischen Umgebung und den Anforderungen an sie, ist es notwendig, die eingesetzte Hard- und Software sowie die entwickelten Prozesse und Tools regelmäßig zu validieren. Dies betrifft sowohl das dLZA-System als Ganzes als auch die innerhalb des Systems eingesetzten Prozesse und Tools. An der TIB wird deshalb in einem jährlichen Intervall eine sogenannte Revalidierung durchgeführt. Außerdem wurde vom ersten Tag des Produktivbetriebes darauf geachtet, dass über ein Exit-Szenario ein Systemwechsel möglich ist. Eine gute Möglichkeit, die Qualität der eigenen Prozesse zu prüfen und zu dokumentieren, ist die Zertifizierung als vertrauenswürdiges Archiv. Die TIB hat diese Qualitätssiegel erworben: 2016 das Data Seal of Approval, ${ }^{17} 2017$ das nestorSiege ${ }^{18}$ und seit 2020 ist sie mit dem Core Trust Seal ${ }^{19}$ zertifiziert.

Trotz des großen Bedarfs ist die digitale Langzeitarchivierung eine Aufgabe, mit der sich nicht jede Institution beschäftigen kann oder will. Denn neben der Bereitstellung einer geeigneten Infrastruktur liegt eine der größten Herausforderungen darin, das für die dLZA erforderliche Know-how aufzubauen und zu halten. Die von der TIB entwickelte Dienstleistung „Preservation-as-a-Service“ greift diesen Bedarf auf. Sie unterstützt ihre Kundinnen und Kunden bei der Aufbereitung und Strukturierung ihrer Daten, führt umfangreiche Prüfroutinen durch und archiviert die ihr übergebenen Inhalte. Sie übernimmt weiterhin das sogenannte Preservation Management, zu dem ein umfangreiches Technology-Watch und CommunityWatch gehört. Verändern sich nun im Laufe der Zeit tech-

17 https://www.coretrustseal.org/about/history/data-seal-of-appro val-synopsis-2008-2018/.

18 https://www.langzeitarchivierung.de/Webs/nestor/DE/Zertifizier ung/nestor_Siegel/siegel.html.

19 https://www.coretrustseal.org/about/. nische Rahmenbedingungen, die Auswirkungen auf die Daten im Archiv haben, werden mögliche Erhaltungsstrategien gemeinsam mit der Kundin oder dem Kunden entwickelt und deren Umsetzung geplant, womit dann ein neuer Erhaltungszyklus beginnt.

\subsection{Open-Access-Transformation}

Open Access - der unbeschränkte und kostenlose Zugang zu wissenschaftlicher Information - ist ein wichtiges Ziel, das die TIB verfolgt. Sie unterstützt die Open-AccessTransformation auf vielfältige Weise. ${ }^{20}$ Ein wichtiges Augenmerk liegt auf der Finanzierbarkeit der Projekte. Wissenschaftlerinnen und Wissenschaftler können sich zu Unterstützungsmöglichkeiten bei der Finanzierung von Open-Access-Kosten beraten lassen. Die TIB übernimmt teilweise die Publikationskosten für neu gegründete OpenAccess-Zeitschriften (zum Beispiel „Quantitative Science Studies“), zeigt auf, welche Formen von Open-Access-Veröffentlichungen es gibt (zum Beispiel in Zeitschriften oder verschiedenen Repositorien) und berät ganz allgemein rund um das Thema Open Access. Darüber hinaus unterstützt die TIB den für Autorinnen und Autoren sowie Leserinnen und Leser kostenlos nutzbaren E-Print-Server arXiv.org ${ }^{21}$ finanziell. ArXiv ist eine bedeutende Informationsquelle und ein wichtiges Medium für die schnelle Veröffentlichung von Forschungsergebnissen in den Fachgebieten Astronomie, Chemie, Informatik, Mathematik und Physik. Die TIB koordiniert weiterhin als Nationale Kontaktstelle in dem von der Deutschen Forschungsgemeinschaft (DFG) geförderten Projekt SCOAP ${ }^{3}-\mathrm{DH}^{22}$ (Sponsoring Consortium for Open Access Publishing in Particle Physics - Deutsche Hochschulen) die Beteiligung deutscher Hochschulen an dem internationalen Pilotprojekt $\mathrm{SCOAP}^{3}$. Das Projekt macht wissenschaftliche Publikationen aus dem Bereich der Hochenergiephysik via Open Access für alle frei zugänglich.

\subsection{Lizenzierung elektronischer Ressourcen}

Lizenzverhandlungen ${ }^{23}$ mit Anbietern, insbesondere mit Verlagen und Fachgesellschaften, sind eine weitere wichtige Aufgabe und Teil der überregionalen Literaturversorgung. Die TIB verhandelt den Erwerb von Nutzungsrechten

20 https://tib.eu/open-access.

$21 \mathrm{http}: / /$ arxiv.org/.

22 https://www.scoap3.de/scoap3-dh.

23 https://tib.eu/lizenzen. 
für digitale Inhalte mit Verlagen und anderen Informationsanbietern für deutsche Hochschulen, Forschungseinrichtungen und wissenschaftliche Bibliotheken, um die Versorgung mit elektronischen Forschungsinformationen nachhaltig $\mathrm{zu}$ verbessern. Die TIB ist eine von acht Bibliotheken, die Allianz-Lizenzen und Nationallizenzen mit Verlagen und anderen Informationsanbietern verhandelt und für die Bereitstellung sorgt. Insgesamt gibt es derzeit 41 laufende Konsortien, in denen die TIB als Verhandlungsführerin fungiert. Insgesamt gab es daran 1050 Teilnahmen, die sich auf 277 Forschungs- und Bildungseinrichtungen verteilten. Die Bibliothek entwickelt Konsortial- und Lizenzmodelle mit den Rechteinhabern, um ihren Nutzerinnen und Nutzern wissenschaftliche Materialien unabhängig von der physischen Form bereitzustellen

\subsection{Das TIB AV-Portal - eine verlässliche Infrastruktur für wissenschaftliche Filme}

Mit dem TIB AV-Portal ${ }^{24}$ hat die TIB eine nutzer- und bedarfsorientierte offene Plattform zum Teilen wissenschaftlicher Videos entwickelt. Der Fokus dieser verlässlichen Infrastruktur liegt auf dem Hosting sowie der Erschließung und Bereitstellung wissenschaftlicher Videos. Das TAVPortal wurde von 2011 bis 2014 von der TIB in Kooperation mit dem Hasso-Plattner-Institut Potsdam entwickelt und ist seit Frühjahr 2014 online verfügbar. Bereitgestellt werden im Portal derzeit mehr als 27000 qualitätsgeprüfte wissenschaftliche Videos (Stand: August 2020). Ein großer Teilbestand umfasst etwa 5000 historische Filme der ehemaligen IWF Wissen und Medien gGmbH. Größtenteils liegen die Videos des AV-Portals unter Creative-CommonsLizenzen vor.

Wesentliche Alleinstellungsmerkmale des Portals sind die Langzeitarchivierung aller Videos sowie die lückenlose Verwendung von Digital Object Identifiern (DOI) und Media Fragment Identifikatoren (MFID). Durch sie werden die langfristig verlässliche Verfügbarkeit sowie die sekundengenaue Referenzierbarkeit der Videos gewährleistet. Darüber hinaus beinhaltet das AV-Portal verschiedene automatisierte Analyseverfahren, durch welche die Videos feingranular und zeitbasiert erschlossen werden. Dabei handelt es sich um eine zeitliche Segmentierung (Erkennung der Einstellungswechsel), eine Keyframe-basierte Texterkennung (Video OCR), die Spracherkennung zur Erstellung von Audiotranskripten, die Annotation mit vordefinierten visuellen Konzepten sowie eine semantische
Analyse und differenzierte Verschlagwortung der Videoinhalte mit circa 65000 fachspezifischen Schlagwörtern der Gemeinsamen Normdatei (GND). Mithilfe dieser Analyseverfahren können Informationen und Inhalte durch semantische und explorative Suchfunktionalitäten („Facettensuche“) präzise lokalisiert, Videos inhaltlich durchsucht und relevante Videoabschnitte segmentgenau identifiziert und aufgerufen werden. Über ein cross-linguales Mapping (deutsch-englisch) der semantischen Wissensbasis lassen sich die Videos darüber hinaus im Portal sprachübergreifend durchsuchen. ${ }^{25}$

Um Dritten eine Nachnutzung der umfangreichen Metadatenbestände des AV-Portals zu ermöglichen, werden sowohl alle bibliografischen Metadaten als auch die automatisch generierten zeitbasierten Metadaten des AVPortals aus Sprach-, Text- und Bilderkennung, die unter Open-Access-Lizenz Creative Commons 1.0 (CCO) stehen, zur Nachnutzung als RDF-Daten (Resource Description Framework) bereitgestellt. Der Datenbestand ist zusätzlich zum DOI mit weiteren Identifikatoren wie ORCID (Open Researcher and Contributor ID), ISIL (International Standard Identifier for Libraries and Related Organisations) und GND-ID (Gemeinsame Normdatei) angereichert, um die Sichtbarkeit und Interoperabilität der Datenbestände zu erhöhen.

\subsection{Digitale Formate}

Ausgewählte Veranstaltungen wie der Digitale Salon oder der „Deutsche Frühling“ in Estland werden live ins Internet gestreamt, damit auch Interessierte, die nicht vor Ort sind, daran teilnehmen können. Anschließend werden die Mitschnitte der Veranstaltungen im AV-Portal der TIB nachgenutzt, womit sie dauerhaft verfügbar sind.

Der in Hannover geplante Bibliothekartag 2020 wurde nach der coronabedingten Absage kurzerhand durch die virtuelle Konferenz \#vBIB20 ersetzt, die der Berufsverband Information Bibliothek (BIB) und die TIB partnerschaftlich veranstaltet haben. Das Ergebnis: Mehr als 3000 Anmeldungen an allen drei Tagen, die fast 70 Stunden Programm mit über 100 Beiträgen zu interessanten und hochaktuellen Themen boten.

Bereits seit einigen Jahren bietet die TIB regelmäßig Webinare und Online-Sprechstunden $\mathrm{zu}$ verschiedenen Themen an. Seit der coronabedingten Schließung der Bibliothek Mitte März 2020 sind umgehend tägliche Webina-

25 Waitelonis et al. (2016). 
re für die Nutzerinnen und Nutzer realisiert worden, in denen sie über aktuelle Entwicklungen, Recherche- und Zugriffsmöglichkeiten informierte und spezifische Fragen der Teilnehmenden beantwortete. Dazu startete eine wöchentliche Telefonsprechstunde zu Citavi, in der es Unterstützung bei der Anwendung des Literaturverwaltungsprogramms gibt.

\section{Wo geht es hin?}

\subsection{Forschungsdaten und Nationale Forschungsdateninfrastruktur (NFDI)}

Wissenschaftliche Forschung generiert eine gigantische und stetig wachsende Menge digitaler Forschungsdaten, die von immenser Bedeutung für die Wissenschaft sind. Sie erhöhen die Transparenz und Nachvollziehbarkeit von Forschungsergebnissen und durch die rasanten technologischen Entwicklungen besteht die Möglichkeit, die Forschungsdaten für weitere Forschungsvorhaben zu nutzen. Die TIB hat sehr früh die globale Dimension und Notwendigkeit erkannt - gemeinsam mit der internationalen Wissenschaftscommunity - Methoden, Maßnahmen und Standards $\mathrm{zu}$ entwickeln, die Forschungsdaten auffindbar, zugänglich, interoperabel und wiederverwendbar machen. So wurde die TIB 2005 die weltweit erste DOI-Registrierungsagentur für Forschungsdaten. Und da Forschung global ist, wurde 2009 der internationale Verein DataCite gegründet. Derzeit werden DataCite-DOIs in 40 Ländern vergeben und für vielfältige Objekttypen wie Forschungsdaten, Software, Videos, Modelle und graue Literatur registriert. Auch nahm der DOI Einzug in die Entwicklungen der European Open Science Cloud (EOSC) und in die Nationale Forschungsdateninfrastruktur (NFDI) - in beiden Initiativen übernimmt die TIB Verantwortung.

Ziel der geplanten NFDI ist die größtmögliche Zugänglichkeit und Erschließung von Forschungsdaten als wertvolle Ressource für die Wissenschaft. Die TIB ist an drei fachwissenschaftlichen Konsortien als Hauptantragsteller beteiligt: dem Fachkonsortium Chemie (NFDI4Chem), ${ }^{26}$ dem Konsortium für Forschungsdaten $\mathrm{zu}$ materiellen und immateriellen Kulturgütern (NFDI4Culture) ${ }^{27}$ und der nationalen Forschungsdateninfrastruktur für die Ingenieurwissenschaften (NFDI4Ing). ${ }^{28}$ Startdatum ist jeweils der

26 http://www.nfdi4chem.de/.

27 www.nfdi4culture.de.

28 www.nfdi4ing.de.
1. Oktober 2020. Auch in den Folgejahren beteiligt sich die TIB noch an weiteren NFDI-Initiativen.

NFDI4Chem besteht aus engagierten Datenproduzenten und -nutzern aus der universitären und außeruniversitären Forschung, Infrastruktureinrichtungen und Fachgesellschaften der Chemie. Ziel ist die Digitalisierung aller Schritte in der chemischen Forschung, um Wissenschaftlerinnen und Wissenschaftler bei der Erfassung, Analyse, nachhaltigen Speicherung, Publikation und Nachnutzung von Forschungsdaten nach den FAIR-Datenprinzipien $\mathrm{zu}$ unterstützen. Ziel von NFDI4Culture ist der Aufbau einer bedarfsorientierten Infrastruktur für Forschungsdaten zu materiellen und immateriellen Kulturgütern in der NFDI. Dazu gehören 2D-Digitalisate von Gemälden, Fotografien und Zeichnungen ebenso wie digitale 3D-Modelle kulturhistorisch bedeutender Gebäude, Denkmäler oder audiovisuelle Daten von Musik-, Film und Bühnenaufführungen. NFDI4Culture adressiert die Bedarfe eines breiten Spektrums an Fachdisziplinen von der Architektur-, Kunst-, Musik- bis hin zur Theater-, Tanz-, Film- und Medienwissenschaft. NFDI4Ing hat zum Ziel, ein gemeinsames Umfeld für ein sicheres Management und die Nachnutzung ingenieurwissenschaftlicher Forschungsdaten $\mathrm{zu}$ schaffen. Dazu wurden innerhalb eines Bottom-up-Prozesses sogenannte Archetypen definiert, welche die verwendeten Forschungsmethoden und -prozesse der ingenieurwissenschaftlichen Teildisziplinen beschreiben. Innerhalb der NFDI4Ing übernimmt die TIB die Rolle eines Infrastrukturund Forschungspartners.

\subsection{Open Research Knowledge Graph (ORKG)}

Als nationales Informationszentrum für Technik und Naturwissenschaften hat die TIB in den vergangenen Jah-ren einen starken Fokus auf die Erforschung und Entwicklung neuer digitaler Methoden für den wissenschaftlichen Informationsaustausch gelegt. Dazu gehören insbesondere der vom europäischen Forschungsrat (ERC) von 2019 bis 2024 mit zwei Millionen Euro geförderte ERC Consolidator Grant ScienceGraph, der Aufbau von mehreren Nachwuchsforschungsgruppen sowie die Entwicklung des Dienstes Open Research Knowledge $\mathrm{Graph}^{29}$ (ORKG). Zukünftige Forschungs-, Innovations- und Entwicklungsarbeiten sollen dabei primär dem weiteren Ausbau und der deutschland-, europa- und weltweiten Etablierung des Dienstes ORKG dienen. Dieser wurde an der TIB seit 2017 entwickelt und ermöglicht es, Forschungserkenntnisse

29 https://tib.eu/orkg. 
in einer semantischen Wissensdatenbank - dem offenen Wissensgraphen - darzustellen, mit anderen wissenschaftlichen Erkenntnissen zu verknüpfen und damit vergleichbar und nachnutzbar zu machen. Dieser Wissensgraph kann, anders als Repositorien von unstrukturierten PDF-Artikeln, effizient durchsucht sowie automatisch analysiert werden sowie Ergebnisse benutzerfreundlich visualisieren. Der ORKG-Dienst ermöglicht dabei eine kollaborative Kuratierung des Wissensgraphs durch Fachwissenschaftlerinnen und Fachwissenschaftler gemeinsam mit Bibliothekarinnen und Bibliothekaren, unterstützt durch Methoden der künstlichen Intelligenz. Im Ergebnis gibt der ORKG Forschenden einen kondensierten Überblick über den Stand der Wissenschaft und ermöglicht auf Basis der reichhaltigen semantischen Repräsentationen gänzlich neue Explorations-, Assistenz- und Visualisierungsunterstützung, die das Potenzial haben, Forschung wesentlich effizienter und effektiver zu gestalten.
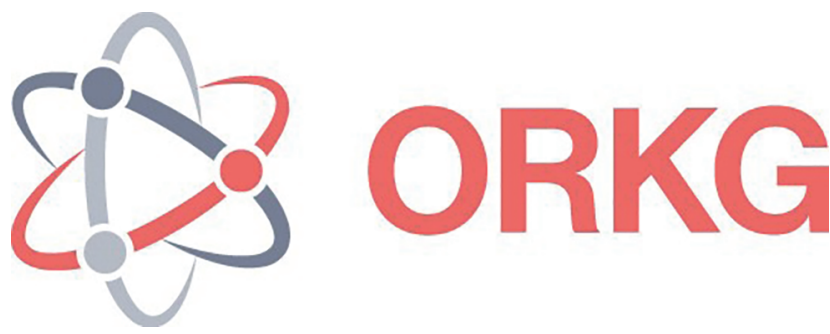

Abb. 3: Logo ORKG

\subsection{Organisation von Daten und Wissen zu Covid-19}

Auch wenn Lebenswissenschaftlerinnen und Lebenswissenschaftler nicht die Kernzielgruppe der TIB sind, wurden an der TIB einige kleinere Beiträge zur Bewältigung der SarsCov2 Pandemie insbesondere bzgl. der Organisation von Daten und Wissen zu Covid-19 erarbeitet:

- Die TIB entwickelt den Open Research Knowledge Graph (ORKG), mit dem Forschungsergebnisse zu Covid-19 strukturiert, organisiert und vergleichbar gemacht werden kann. So können Überschneidungen erkannt und auf jedem der Teilgebiete der jeweils aktuelle Stand erfasst werden.

- Außerdem wird an der TIB die Daten-Pipeline „Knowledge4Covid-19“ zur Erfassung und Zusammenführung von Covid-19-Forschungsergebnissen implementiert. Dabei werden Covid-19-Ergebnisse mit bereits existierenden Forschungsergebnissen verknüpft und über maschinelle Lernverfahren ausgewertet, um neue Erkenntnisse zu gewinnen.
- Als weitere Initiative stellt das Open Science Lab durch die Book-Sprint-Initiative dringend benötigte Literatur zum Umgang mit Covid-19 für den Gesundheitssektor und die Fachöffentlichkeit zur Verfügung. So wird der wichtige Transfer von Forschungsergebnissen in die operativen Bereiche der Gesundheitsversorgung und Prävention realisiert.

- Die TIB nahm am \#EUvsVirus Pan-European Hackathon teil, veranstaltet von der Europäischen Kommission, um die Zivilgesellschaft, Innovatoren, Partner und Investoren in ganz Europa für innovative Lösungen in Bezug auf die Coronapandemie zu entwickeln.

\section{Schlusswort}

Zusammenfassend lässt sich sagen, dass die Verzahnung von Forschung, Innovation und bibliothekarischen Diensten inzwischen in die DNA der TIB übergegangen ist. Wir hoffen, damit auch in Zukunft einen Beitrag zur Weiterentwicklung des Bibliothekswesens und zur Lösung von Herausforderungen unserer Nutzerinnen und Nutzer leisten zu können. Diese Herausforderungen wollen wir gemeinsam mit Partnern, der Bibliothekscommunity sowie weiteren Mitstreiterinnen und Mitstreitern in Projekten wie dem Open Research Knowledge Graph oder der Nationalen Forschungsdateninfrastruktur angehen.

\section{Literaturverzeichnis}

Schlitt, Gerhard (1981): Universitätsbibliothek und Technische Informationsbibliothek. In: Universität Hannover 1831-1981. Festschrift zum 150-jährigen Bestehen der Universität, hg. v. Rita Seidel im Auftrag des Präsidenten, 464-67. Hildesheim: Olms.

Tempel, Bernhard (2020): Lebendige Kooperation. Das Hannoversche Online-Bibliothekssystem (HOBSY). In: BuB - Forum Bibliothek und Information, 72 (6), 359-61.

Vollers, Hinrich (1983): Die Niedersächsische Landesbibliothek als Universitätsbibliothek. In: ABI-Technik, 3 (2), 91-98.

Vollers, Hinrich (1995): Kooperative Literaturversorgung der Universität Hannover. 25 Jahre Niedersächsische Landesbibliothek als Universitätsbibliothek. In: mb. Mitteilungsblatt der Bibliotheken in Niedersachsen und Sachsen-Anhalt, 96, 4-23.

Waitelonis J.; Plank M.; Sack H. (2016): TIB|AV-Portal: Integrating Automatically Generated Video Annotations into the Web of Data. In: Research and Advanced Technology for Digital Libraries, ed. by N. Fuhr, L. Kovács, T. Risse and W. Nejdl. TPDL 2016. (Lecture Notes in Computer Science, 9819). Springer: Cham. Available at https://doi.org/10.1007/978-3-319-43997-6_37. 


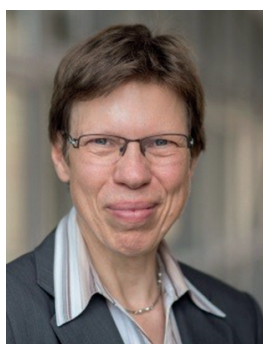

Dr. Irina Sens

TIB - Leibniz-Informationszentrum Technik und Naturwissenschaften

Stellvertretende Direktorin der TIB

Welfengarten $1 \mathrm{~B}$

D-30167 Hannover

irina.sens@tib.eu

https://orcid.org/0000-0001-9190-8628

(C) TIB/C. Bierwagen

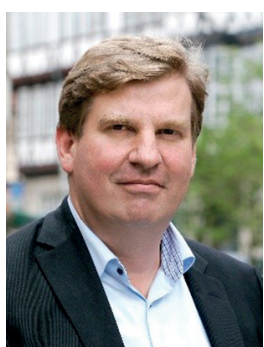

\section{Prof. Dr. Sören Auer}

TIB - Leibniz-Informationszentrum Technik und Naturwissenschaften

Direktor der TIB

Welfengarten $1 \mathrm{~B}$

D-30167 Hannover

auer@tib.eu

https://orcid.org/0000-0002-0698-2864

(C) TIB/C. Behrens

\section{Thomas Bähr}

TIB - Leibniz-Informationszentrum Technik und

Naturwissenschaften

Leitung Bestandserhaltung und Langzeitarchivierung

Welfengarten $1 \mathrm{~B}$

D-30167 Hannover

thomas.baehr@tib.eu

\section{Dr. Sandra Niemeyer}

TIB - Leibniz-Informationszentrum Technik und Naturwissenschaften

Kommunikation und Marketing

Welfengarten $1 \mathrm{~B}$

D-30167 Hannover

sandra.niemeyer@tib.eu

\section{Margret Plank}

TIB - Leibniz-Informationszentrum Technik und Naturwissenschaften

Leitung Lab Nicht-Textuelle Materialien

Welfengarten $1 \mathrm{~B}$

D-30167 Hannover

margret.plank@tib.eu

\section{Philip Schrenk}

Leitung Lab Nicht-Textuelle Materialien

TIB - Leibniz-Informationszentrum Technik und Naturwissenschaften

Welfengarten $1 \mathrm{~B}$

D-30167 Hannover

philip.schrenk@tib.eu 